INPLASY

PROTOCOL

To cite: Yan et al. Combination of pulsed radiofrequency with continuous radiofrequency thermocoagulation for patients with trigeminal neuralgia: $A$ meta-analysis of randomized controlled trials. Inplasy protocol 202220100. doi: 10.37766/inplasy2022.2.0100

Received: 23 February 2022

Published: 23 February 2022

Corresponding author:

Tong-qing Xue

myd11033@163.com

Author Affiliation:

Huaian Hospital of Huaian City

Support: N/A.

Review Stage at time of this submission: Study completed.

Conflicts of interest:

None declared.

\section{Combination of pulsed radiofrequency with continuous radiofrequency thermocoagulation for patients with trigeminal neuralgia: A meta-analysis of randomized controlled trials}

Yan, CY1; Zhang, QX2; Liu, C3; Yang, J4; Bian, H5; Zhu, J6; Xue, $\mathrm{TQ}^{7}$.

Review question / Objective: To compare the treatment effectiveness of combined Continuous radiofrequency thermocoagulation (CRF) and pulsed radiofrequency (PRF) versus CRF alone for patients with trigeminal neuralgia by using meta-analytic approach.

Condition being studied: The previous studies found that combined therapy of Continuous radiofrequency thermocoagulation (CRF) with Pulsed radiofrequency (PRF) in the treatment of trigeminal neuralgia was more beneficial than CRF alone, which combined therapy was associated with higher incidence of cure rate, lower facial numbness score, and higher quality of life. There are also several studies suggested combined therapy of CRF with PRF may not superior to CRF alone in improvement of pain symptom, incidence of cure rate, and complications (e.g., masticatory muscle weakness). It is obvious that still existed a controversy in whether the use combined of CRF with PRF could yields additional effects over CRF alone for patients with trigeminal neuralgia remains unclear. We therefore conducted a systematic review and meta-analysis based on randomized controlled trials (RCTs) to determine the treatment effectiveness between combined CRF with PRF and CRF for patients with trigeminal neuralgia.

INPLASY registration number: This protocol was registered with the International Platform of Registered Systematic Review and Meta-Analysis Protocols (INPLASY) on 23 February 2022 and was last updated on 23 February 2022 (registration number INPLASY202220100).

\section{INTRODUCTION}

Review question / Objective: To compare the treatment effectiveness of combined Continuous radiofrequency thermo- coagulation (CRF) and pulsed radiofrequency (PRF) versus CRF alone for patients with trigeminal neuralgia by using meta-analytic approach. 
Rationale: PubMed, EmBase, Cochrane library, and China National Knowledge Infrastructure were systematically searched for RCTs met the inclusion criteria throughout October 2020. The investigated outcomes including cure rate, pain score, facial numbness score, quality of life, and potential complications. The random-effects model was applied to calculate the pooled effect estimates.

Condition being studied: The previous studies found that combined therapy of Continuous radiofrequency thermocoagulation (CRF) with Pulsed radiofrequency (PRF) in the treatment of trigeminal neuralgia was more beneficial than CRF alone, which combined therapy was associated with higher incidence of cure rate, lower facial numbness score, and higher quality of life. There are also several studies suggested combined therapy of CRF with PRF may not superior to CRF alone in improvement of pain symptom, incidence of cure rate, and complications (e.g., masticatory muscle weakness). It is obvious that still existed a controversy in whether the use combined of CRF with PRF could yields additional effects over CRF alone for patients with trigeminal neuralgia remains unclear. We therefore conducted a systematic review and meta-analysis based on randomized controlled trials (RCTs) to determine the treatment effectiveness between combined CRF with PRF and CRF for patients with trigeminal neuralgia.

\section{METHODS}

Search strategy: The Preferred Reporting Items for Systematic Reviews and MetaAnalyses was used to guide the performing and reporting of this study. Study designed as RCT and compared the efficacy and safety of combined therapy with CRF alone for patients with trigeminal neuralgia was eligible in this study, and the publication language and status were not restricted. We systematically searched the databases of PubMed, EmBase, Cochrane library, and China National Knowledge Infrastructure for eligible studies and the following search terms were used by text words and Medical
Subject Headings: "trigeminal neuralgia," "TN," "semilunar ganglion," "gasserian ganglion," "oval foramen," "pulsed," "continuous," "percutaneous," "conventional," "radiofrequency thermocoagulation," "radiofrequency ablation." We also reviewed the reference lists of retrieved studies to identify any new trial met the inclusion criteria through manually. This study did not contain any patients' data, and ethics approval and consent to participate are not applicable. Study was included if they met: (1) Patients: trigeminal neuralgia; (2) Intervention: combined CRF with PRF; (3) Control: CRF alone; (4) Outcome: the primary outcome is cure rate, pain score, facial numbness score, quality of life, numbness, masticatory muscle weakness, corneal palsy are included secondary outcomes; and (5) Study design: all studies had to have RCT design. The study selections were independently performed by 2 reviewers, and inconsistencies between reviewers was settled by discussion until a consensus was reached.

Participant or population: A total of $\mathbf{3 1 7}$ patients with trigeminal neuralgia were recruited from included studies, and the sample size ranged from 31 to 90 . Four trials were conducted in China, and the remaining 1 trial was conducted in Egypt.

Intervention: Continuous radiofrequency thermocoagulation (CRF) with Pulsed radiofrequency (PRF).

Comparator: Continuous radiofrequency thermocoagulation (CRF) alone.

Study designs to be included: Randomized controlled trials (RCTs).

Eligibility criteria: (1) Patients: trigeminal neuralgia; (2) Intervention: combined CRF with PRF; (3) Control: CRF alone; (4) Outcome: the primary outcome is cure rate, pain score, facial numbness score, quality of life, numbness, masticatory muscle weakness, corneal palsy are included secondary outcomes; and (5) Study design: all studies had to have RCT design. 
Information sources: Databases: PubMed, EmBase, Cochrane library, and China National Knowledge Infrastructure Contact with authors: All dataset was extracted from article without contact with authors. Trial register: All studies are presented trial registers in all articles.

Main outcome(s): Cure rate: BNI pain scale, excellent level, pain free with no medication.

Additional outcome(s): 1. Pain Score: Visual Analogue Scale/Score; 2. Facial numbness score: Facial numbness score scale; 3. Quality of life: EQ-5D, Short Form-36 questionnaire (SF-36); 4. Complications: Numbness, masticatory muscle weakness and Corneal palsy.

Data management: Two reviewers independently performed data collection and quality assessment, and any disagreement between reviewers was resolved by an additional reviewer reading the original article.

Quality assessment / Risk of bias analysis: The quality of each trial was assessed by using the version 2 of the Cochrane risk-ofbias tool (RoB2), which bias domains contains bias arising from the randomization process (low risk/some concerns/high risk), bias due to deviations from intended interventions (low risk/some concerns/high risk), bias due to missing outcome data (low risk/some concerns/ high risk), bias in measurement of the outcome (low risk/some concerns/high risk), bias in selection of the reported result (low risk/some concerns/high risk) and overall bias (low risk/some concerns/high risk).

Strategy of data synthesis: The effectiveness of combined CRF with PRF versus CRF alone for patients with trigeminal neuralgia were assigned as categorical and continuous outcomes, then odds ratio (OR) and weighted mean difference (WMD) with 95\% confidence interval $(\mathrm{Cl})$ were calculated for each trial before data pooling. After this, the pooled effect estimates between combined CRF with PRF and CRF alone were calculated by using the random-effects model. 12 and $Q$ statistic were used to assess the heterogeneity across included studies, and significant heterogeneity was defined as $\mathbf{1 2}$ $>\mathbf{5 0 . 0 \%}$ or $\mathrm{P}<0.10$.

Subgroup analysis: Subgroup analyses were also conducted for treatment effectiveness based on follow-up duration. Publication bias for efficacy outcomes were evaluated by using Funnel plots, Egger and Begg test results.

Sensitivity analysis: We used the version 2 of the Cochrane risk-of-bias tool (RoB2) to delete the low quality of studies and analysis to ensure the stability of all present results.

Language: English and Chinese.

Country(ies) involved: China and Egypt.

Keywords: Laser Coagulation; Pulse Radiolysis; Facial Paralysis; Meta-Analysis

Contributions of each author:

Author 1 - Cong-yang Yan.

Email: 290981453@qq.com

Author 2 - Qian-xi Zhang.

Email: 13852301610@139.com

Author 3 - Cheng Liu.

Email: 1450493568@qq.com

Author 4 - Jia-li Yang.

Email: 714797761@qq.com

Author 5 - Hu Bian.

Email: 13770391949@126.com

Author 6 - Jun Zhu.

Email: zhujun320634@163.com

Author 7 - Tong-qing Xue.

Email: myd11033@163.com 